EPJ Web of Conferences 59, 05014 (2013)

DOI: $10.1051 /$ epjconf/20135905014

(C) Owned by the authors, published by EDP Sciences, 2013

\title{
Vlasov simulations of self generated strong magnetic fields in plasmas and laser-plasma interaction
}

\author{
A. Inglebert ${ }^{1}$, A. Ghizzo ${ }^{1, a}$, T. Reveille ${ }^{1}$, D. Del Sarto ${ }^{1}$, P. Bertrand ${ }^{1}$ \\ and F. Califano ${ }^{2}$ \\ 1 IJL UMR 7198, University of Lorraine, BP. 239, 54506 Vandoeuvre-les-Nancy, France \\ 2 Physics Department, University of Pisa, Pisa, Italy
}

\begin{abstract}
A new formulation based on Hamiltonian reduction technique using the invariance of generalized canonical momentum is introduced for the study of relativistic Weibel-type instability. An example of application is given for the current filamentation instability resulting from the propagation of two counterstreaming electron beams in the relativistic regime of the instability. This model presents a double advantage. From an analytical point of view, the method is exact and standard fluid dispersion relations for Weibel or filamentation instabilies can be recovered. From a numerical point of view, the method allows a drastic reduction of the computational time. A 1D multi-stream Vlasov-Maxwell code is developed using such dynamical invariants in the perpendicular momentum space. Numerical comparison with a full VlasovMaxwell system has also been carried out to show the efficiency of this reduction technique.
\end{abstract}

\section{INTRODUCTION}

As a fundamental issue, the Weibel instability [1] or the current filamentation instability [2-5] are able to generate a magnetic field by extracting the free energy from an anisotropy velocity distribution in an unmagnetized plasma. The propagation of a hot electron beam in a plasma induces a return current in the background plasma to keep current neutralization of the beam-plasma system, resulting in the current filamentation instability (CFI). Such a scenario is also met and relevant to the concept of the fast ignitor [6] of laser inertial confinement fusion.

We develop here a multi-stream model, based on a Hamiltonian reduction technique. For a onedimensional spatial plasma, exact transverse canonical invariants are used to construct a broad class of exact nonlinear solutions of the Vlasov-Maxwell system [7].

The paper is organized as follows. In section 2 we present our kinetic multi-stream model as derived from the Vlasov-Maxwell system. Then we deduce the (fluid) dispersion relation in the relativistic regime in section 3. Numerical comparisons of our kinetic multi-stream Vlasov model with the standard 1D2V full-kinetic Vlasov-Maxwell are shown in section 4 for a symetric case of CFI and finally section 5 is reserved for the conclusions and discussion.

\section{THE MUTI-STREAM VLASOV-MAXWELL MODEL}

In this section we will restrict to plane waves propagating along the $x$ direction and assume that ions are kept fixed. We have to solve a Vlasov equation for a $4 \mathrm{D}$ electron distribution function

\footnotetext{
${ }^{a}$ e-mail: Alain.Ghizzo@ijl.nancy-universite.fr
}

This is an Open Access article distributed under the terms of the Creative Commons Attribution License 2.0, which permits unrestricted use, distribution, and reproduction in any medium, provided the original work is properly cited. 
$F(x, \mathbf{p}, t)$ :

$$
\frac{\partial F}{\partial t}+\frac{p_{x}}{m_{e} \gamma} \frac{\partial F}{\partial x}+e\left(\mathbf{E}+\frac{\mathbf{p} \times \mathbf{B}}{m_{e} \gamma}\right) \frac{\partial F}{\partial \mathbf{p}}=0
$$

where the (squared) Lorentz factor has the conventional form: $\gamma^{2}=1+p^{2} / m_{e}^{2} c^{2}$.

Let us consider the Hamiltonian of one particle in the electromagnetic field $(\mathbf{E}, \mathbf{B})$ in the relativistic regime, $H=m_{e} c^{2}(\gamma-1)+e \phi(x, t)$ with the Lorentz factor: $\gamma=\left[1+\left(\mathbf{P}_{c}-e \mathbf{A}_{\perp}(x, t)\right)^{2} / m_{e}^{2} c^{2}\right]^{1 / 2}$ where $\phi$ and $\mathbf{A}=\mathbf{A}_{\perp}$ denote respectively, the electrostatic potential and the vector potential. We have here introduced the canonical momentum $\mathbf{P}_{c}$ connected to the particle momentum $\mathbf{p}$ by: $\mathbf{P}_{c}=\mathbf{p}+e \mathbf{A}_{\perp}$. In the Coulomb gauge $\operatorname{div} \mathbf{A}=0, \mathbf{A}=\mathbf{A}_{\perp}$. Thus the Hamilton equation $d \mathbf{P}_{c} / d t=-\partial H / \partial \mathbf{q}$ can be written in the longitudinal and perpendicular directions as: $d P_{c x} / d t=-\partial H / \partial x$ and $d \mathbf{P}_{c \perp} / d t=$ $-\partial H / \partial \mathbf{q}_{\perp}=0$ since the Hamiltonian $H$ does not depend of the transverse spatial coordinates. Explicitly, it is the invariance of the perpendicular canonical momentum $\mathbf{P}_{c \perp}$ which allows us to built the multi-stream model using the Hamiltonian formalism.

Without loss of generality, we can consider a plasma, initially prepared so that particles are divided into $N$ bunches of particles, each "stream" $j$ (with $j=1, \ldots, N$ ) having the same initial perpendicular momentum $\mathbf{P}_{c \perp}=\mathbf{C}_{j}=$ const. It is then possible to define, for each stream $j$ a distribution function $f_{j}\left(x, p_{x}, t\right)$ which must satisfy the Vlasov equation:

$$
\frac{\partial f_{j}}{\partial t}+\frac{p_{x}}{m_{e} c} \frac{\partial f_{j}}{\partial x}+\left(e E_{x}-\frac{1}{2 m_{e} \gamma_{j}} \frac{\partial}{\partial x}\left(\mathbf{C}_{j}-e \mathbf{A}_{\perp}\right)^{2}\right) \frac{\partial f_{j}}{\partial p_{x}}=0, \quad \text { for } \quad j=1, \ldots, N .
$$

The Lorentz factor $\gamma_{j}$, for the particle bunch $j$, is : $\gamma_{j}=\left[1+p_{x}^{2} / m_{e}^{2} c^{2}+\left(\mathbf{C}_{j}-e \mathbf{A}_{\perp}(x, t)\right)^{2} / m_{e}^{2} c^{2}\right]^{1 / 2}$.

Therefore the full distribution function $F\left(x, p_{x}, \mathbf{p}_{\perp}, t\right)$ can now be written in a sum of a Dirac distributions of kind :

$$
F\left(x, p_{x}, \mathbf{p}_{\perp}, t\right)=\sum_{j=1}^{N} f_{j}\left(x, p_{x}, t\right) \delta\left(\mathbf{p}_{\perp}-\left(\mathbf{C}_{j}-e \mathbf{A}_{\perp}(x, t)\right)\right)
$$

thus, reducing the 4D phase space into a $2 \mathrm{D}$ one (plus $N$ values for the corresponding $\mathbf{C}_{j}$ ).

Our kinetic multi-stream model is given by $N$ reduced Vlasov equations of type (2) for each stream population $j$, coupled self-consistently with the Maxwell-Gauss and the Maxwell-Ampere equations through the source terms $n(x, t)=\sum_{j} n_{j}$ and the total perpendicular current $\mathbf{J}_{\perp}=\sum_{j} \mathbf{J}_{\perp j}$ respectively.

\section{THE MULTI-STREAM MODEL IN THE FLUID APPROXIMATION AND STANDARD DISPERSION RELATION}

Our model is applied for the calculation of the standard dispersion relation of the Weibel-type instabilities. For instance the case $N=2$ is straightforward and corresponds exactly to the case of the current filamentation instability.

By considering the successive moments of the Vlasov equation (2), a multi-fluid model can be then obtained. By assuming a linearly polarized electromagnetic wave, for each stream $j$, the continuity and Euler equations can be derived:

$$
\begin{gathered}
\frac{\partial n_{j}}{\partial t}+\frac{\partial}{\partial x}\left(\frac{n_{j} u_{j}}{m_{e} \tilde{\gamma}_{j}}\right)=0 \\
\frac{\partial u_{j}}{\partial t}+\frac{u_{j}}{m_{e} \tilde{\gamma_{j}}} \frac{\partial u_{j}}{\partial x}=e E_{x}-\frac{1}{2 m_{e} \tilde{\gamma}_{j}} \frac{\partial}{\partial x}\left(C_{j}-e A_{y}\right)^{2} .
\end{gathered}
$$


The Lorentz factor, in the fluid approximation becomes: $\tilde{\gamma}_{j}=\left[1+u_{j}^{2} / m_{e}^{2} c^{2}+\left(C_{j}-e A_{y}(x, t)\right)^{2}\right.$ $\left./ m_{e}^{2} c^{2}\right]^{1 / 2}$.

We consider an expansion around an equilibrium characterized by a mean density $n_{0 j}$ and a mean longitudinal momentum $u_{0 j}=0$ and assume $\sum_{j=1}^{N} n_{0 j}=n_{0}$ and $\sum_{j=1}^{N} \frac{C_{j} n_{0 j}}{\Gamma_{0 j}}=0$, corresponding at zero order to the neutrality condition and to the requirement that the initial net current is zero, respectively. $\Gamma_{0 j}$ takes into account, at the zero-order, the pure transverse contribution in the expression of the Lorentz factor: $\Gamma_{0 j}=\sqrt{1+C_{j}^{2} / m_{e}^{2} c^{2}}$. By linearizing and performing a time/space Fourier transform, we get the dispersion relation for Weibel-types instabilities:

$$
\left(1-\sum_{j=1}^{N} \frac{\omega_{p j}^{2}}{\omega^{2} \Gamma_{0 j}}\right)\left\{-\omega^{2}+k^{2} c^{2}+\sum_{j=1}^{N} \frac{\omega_{p j}^{2}}{\Gamma_{0 j}^{3}}+\frac{k^{2} c^{2}}{\omega^{2}} \sum_{j=1}^{N} \frac{\omega_{p j}^{2}}{\Gamma_{0 j}^{3}} \frac{C_{j}^{2}}{m_{e}^{2} c^{2}}\right\}=-\frac{k^{2} c^{2}}{\omega^{2}}\left(\sum_{j=1}^{N} \frac{\omega_{p j}^{2}}{\Gamma_{0 j}^{2}} \frac{C_{j}}{m_{e} c}\right)^{2}
$$

The term $\omega_{p j}^{2}=n_{0 j} e^{2} / m \varepsilon_{0}$ is the "plasma frequency" of the particle bunch $j$. The analytical formulation of the linear dispersion relation, Eq. (6), as a discrete summation over an assembly of streams provides a general and exact approach able to take into account any anisotropy of the distribution function, even in the relativistic regime, in agreement with previous results in ref. [8].

\section{NUMERICAL COMPARISON BETWEEN THE 1D2V FULL KINETIC VERSION AND THE REDUCED VLASOV MODEL}

We have checked the efficiency and accuracy of our method in the case of the current filamentation instability, which corresponds here to take only two streams in the reduced model (R-model hereafter). We have performed numerical simulations of the $1 \mathrm{D} 2 \mathrm{~V}$ full kinetic Vlasov equation ( $\mathrm{V}$-model) given by Eq. (1) and then of the 1D reduced Vlasov equations (2), using a phase space code based on a splitting method and semi-lagrangian scheme [9-11] of the Vlasov equation. Both Vlasov models were solved in parallel with the Maxwell's equations.

The initial distribution condition is composed of a two Maxwellians with beam momenta centered at $\mathbf{p}_{01} / m_{e} c=-0.9 \mathbf{e}_{y}$ and $\mathbf{p}_{02} / m_{e} c=0.9 \mathbf{e}_{y}$. Note that we are in a semi-relativistic regime. We have chosen a symetric case corresponding to beam densities of $n_{01}=n_{02}=0.5 n_{0}$. Here we focus the attention on purely transverse initial perturbation (on the magnetic $B_{z^{-}}$component) with wave vector of type $\mathbf{k}_{0}=k_{0} \mathbf{e}_{x}$ along the $x$-direction (here the longitudinal direction), perpendicular to the two counterstreaming electron beams. We perturb the system using a small perturbation of $e B_{0} / m_{e} \omega_{p}=10^{-4}$ and we take $k_{0} c / \omega_{p}=1$. Electron plasma temperature is chosen to $T_{e}=2 \mathrm{keV}$ in both the $p_{x}$ and $p_{y}$ direction in the $\mathrm{V}$-model while, in the R-model, only the $p_{x}$ direction has a non zero temperature. We keep the same temperature in the R-model. The phase space sampling used here in the 1D2V version is $N_{x} N_{p_{x}} N_{p_{y}}=256 \times 257^{2}$, i.e. $1.69 \times 10^{7}$ grid points or "particles". The time step used in both simulation is here $\Delta t \omega_{p}=0.003$. The phase space sampling, used in the presented simulation is somewhat higher with $N_{x} N_{p_{x}}$ given by $513^{2}$ grid points.

First we have resolved, for the corresponding physical parameters of simulation, the dispersion relation (6) in order to obtain an accurate estimation of the growth rate of the instability in the linear phase. The corresponding result is shown in the left panel in Fig. 1: we have plotted the growth rate $\eta_{t h} / \omega_{p}$ as a function of the wave vector $k c / \omega_{p}$ using two streams, i.e. with the data $N=2$ in Eq. (6). On the right panel, in Fig. 1, we have represented the time evolution of the magnetic energy, in a logarithmic scale. As expected the magnetic mode is unstable and grows with a linear growth rate of $\eta_{\text {num }} / \omega_{p} \simeq 0.448$, in good agreement with the theoretical value of $\eta_{t h} / \omega_{p}=0.45$, for a value of $k c / \omega_{p}=1$.

In Fig. 2, we show the electron distribution in the $x-p_{x}$ (left panel) phase space, for the V-model when the instability starts to saturate. The same $x-p_{x}$ phase space dynamic, described by the R-model 

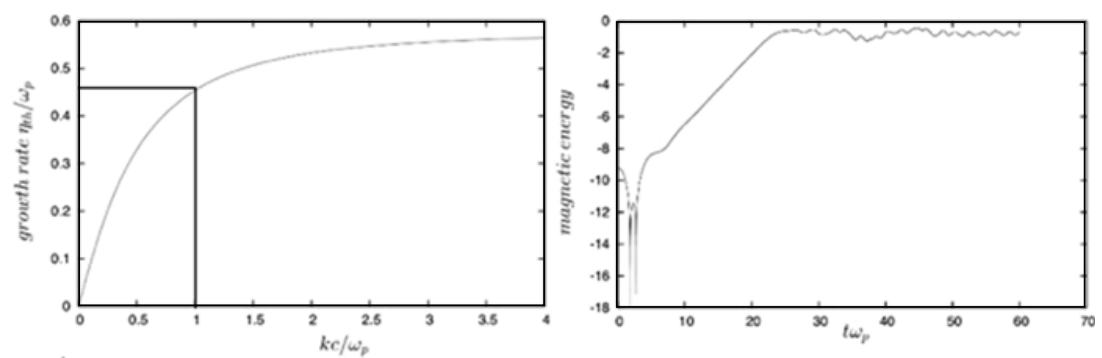

Figure 1. On left panel, the growth rate as a function of the wave vector obtained by resolving the dispersion relation $\eta_{t h} / \omega_{p}=0.45$, for $k c / \omega_{p}=1$. On the right panel, time evolution of the magnetic energy, in a logarithmic scale $\eta_{\text {num }} / \omega_{p} \simeq 0.448$, in good agreement with the theoretical value.
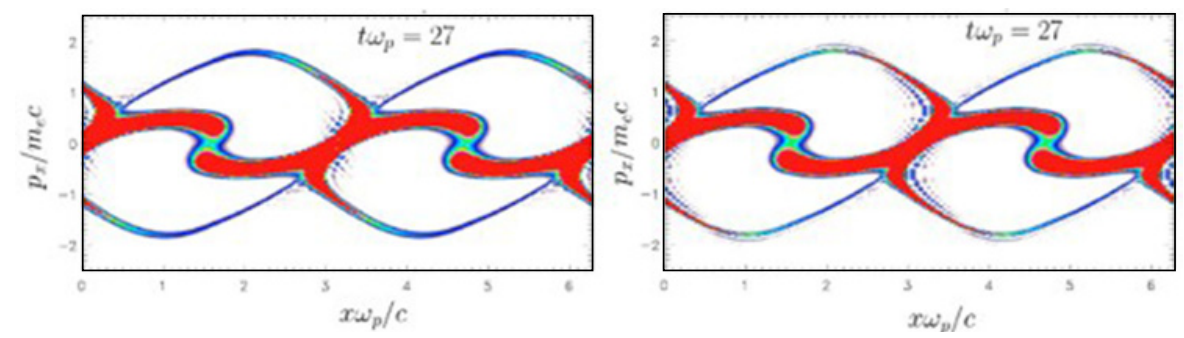

Figure 2. On the left panel, phase space behavior of the electron distribution function in the $x-p_{x}$ obtained from the V-model. On the right panel, $x-p_{x}$ obtained from the R-model.

using the sum of the two streams $\sum_{j} f_{j}$, is shown in the right panel at the same time of the one obtained from the V-model. These figures show that the dynamics is correctly described by the R-model where only two streams are considered.

\section{CONCLUSION}

This paper is an attempt to reduce the dimension of the phase space while keeping the high level of accuracy afforded by Vlasov codes. This reduction technique uses the Hamiltonian formulation and the existence of dynamical invariants. Thus detailed comparison of numerical results obtained by using a reduced 1D model have been carried out with those obtained with a full kinetic and relativistic $1 \mathrm{D} 2 \mathrm{~V}$ version. Good agreement provides full support for the 1D electromagnetic and relativistic Vlasov code which runs considerably faster than the $1 \mathrm{D} 2 \mathrm{~V}$ code. However, within the capacity of supercomputers, a full kinetic 2D version (i.e. with 4D dimensional phase space) is now available and can be of great interest when the coupling of Weibel-type instability with the purely electrostatic two-stream instability must be taken into account.

The authors are indebted to the IDRIS computational center, Orsay, France, for computer time allocation on their computers.

\section{References}

[1] E.W. Weibel, Phys. Rev. Lett. 2, 83 (1959)

[2] A.R. Bell, J. R. Davies, S. Guerin, H. Ruhl, Plasma Phys. Control. Fus. 39, 653 (1997) 


\section{IFSA 2011}

[3] B.D. Fried, Phys. Fluids 2, 337 (1959)

[4] F. Califano, F. Pegoraro, S.V. Bulanov, A. Mangeney, Phys. Rev. E 57, 7048 (1998)

[5] F. Califano, R. Prandi, F. Pegoraro, S.V. Bulanov, J. Plasma Phys. 60, 331 (1998)

[6] M. Tabak, J. Hammer, M.E. Glinsky, W.L. Kruer, S.C. Wilks, J. Woodworth, E.M. Campbell, M.D. Perry, R.J. Mason, Phys. Plasmas 1, 166 (1994)

[7] A. Inglebert, A. Ghizzo, T. Reveille, D. Del Sarto, P. Bertrand, F. Califano, EuroPhys. Lett. 95, 45002 (2011)

[8] F. Pegoraro, S.V. Bulanov, F. Califano, M. Lontano Phys. Scripta T63, 262 (1996)

[9] F. Huot, A. Ghizzo, P. Bertrand, E. Sonnendrucker, O. Coulaud J. Comput. Phys. 185, 512 (2003)

[10] A. Ghizzo, F. Huot, P. Bertrand, J. Comput. Phys. 186, 47 (2003)

[11] M.L. Begue, A. Ghizzo, P. Bertrand, J. Comput. Phys. 151, 458 (1999) 